\title{
Development of the Japanese version of the Minneapolis-Manchester Quality of Life Survey of Health - Adolescent Form (MMQL-AF) and investigation of its reliability and validity
}

\author{
Makiko Koike ${ }^{1,2^{*}}$, Hiroki Hori ${ }^{3}$, Takeshi Rikiishi ${ }^{4}$, Akira Hayakawa ${ }^{5}$, Naoko Tsuji ${ }^{6}$, Tsukasa Yonemoto ${ }^{7}$, Hideko Uryu ${ }^{8}$
} and Eisuke Matsushima ${ }^{1}$

\begin{abstract}
Background: There are very few reliable and valid measures in Japan assessing health-related quality of life (HRQOL) in children with cancer. The present study aimed to develop a Japanese version of the Minneapolis-Manchester Quality of Life Survey of Health Adolescent Form (MMQL-AF), which is a measure for assessing the HRQOL of childhood cancer survivors, and investigate its reliability and validity.

Methods: Participants were 141 children with cancer who had been off therapy for more than one year and 183 healthy controls. The reliability and internal consistency of the measure were assessed through test-retest methods using Cronbach's coefficient alpha and intra-class correlation coefficients (ICCS). For validation of the measure, factorial validity, concurrent validity using the Japanese version of PedsQL 4.0 Generic Core Scales (PedsQL-J), and discriminant validity using comparisons between children with cancer and healthy controls were investigated.

Results: Of the 46 items in the original version, 44 items were determined to comprise the Japanese version of the MMQL-AF. Cronbach's coefficient alphas for each subscale were high ranging from 0.83 to 0.89 . Test-retest reliability ranged between ICC 0.79 to 0.96 . Investigation of concurrent validity using the PedsQL-J demonstrated strong correlations in physical functions and moderate correlations for other factors. A significant difference was observed between children with cancer and healthy controls.
\end{abstract}

Conclusions: Thus, the Japanese version of the MMQL-AF served as a self-evaluation questionnaire that allowed for practical, comprehensive, and multidimensional measurement of HRQOL specific to childhood cancer survivors.

Keywords: Childhood cancer, Survivor, Health-related quality of life

\section{Background}

The treatment of childhood cancer has made remarkable progress with multidisciplinary treatment. A cure can be expected in more than $70-80 \%$ of children who have been diagnosed with childhood cancer [1-3]. However, many children are facing various late effects associated with the treatment directly and/or indirectly through

\footnotetext{
* Correspondence: mkoike@mejiro.ac.jp

${ }^{1}$ Section of Liaison Psychiatry and Palliative Medicine, Graduate School of Tokyo Medical and Dental University, 1-5-45 Yushima, Bunkyo-ku, Tokyo 113-8519, Japan

${ }^{2}$ Graduate School of Psychology, Mejiro University, 4-31-1 Nakaochiai, Shinjuku-ku, Tokyo 161-8539, Japan

Full list of author information is available at the end of the article
}

long-term treatment, including chemotherapy, radiotherapy, and surgical therapy, in the course of overcoming childhood cancer, in addition to the disorders caused by the disease itself [4-6]. Late effects include impaired growth and development, disorders of the endocrine system such as gonadal dysfunction and hypothalamopituitary disorders accompanying impaired growth and development, cardiac/renal dysfunction, secondary cancer associated with treatment, intellectual impairment, and psychological disorders. The types and symptoms of late effects differ depending on the disease, therapy, the age the patient receiving the therapy, and sex; thus, any late effects can be a factor deteriorating the quality of life 
(QOL) of the children $[7,8]$. Even in children who recovered without physical late effects, many difficulties with the aspect of psychological or social QOL have been reported. For example, when entering school, employment, and into a marriage, they tend to suffer a psychological burden and social disadvantage, thus the decrease in QOL in these patients is a concern [9-11].

Developmental psychologists have noted that self-esteem has been a significant factor influencing identity formation during adolescence [12]. Furthermore, developmental psychologists have also conducted studies examining the association between QOL and self-esteem in child cancer survivors during adolescence [13-16].

With these points of view, it is essential to construct a follow-up support system focusing on maintaining or improving long-term QOL, including the process of growth and development after a cure, as well as during treatment, for children with cancer [17-19]. Thus, a study on healthrelated quality of life (HRQOL) in childhood cancer survivors is a top priority in order to optimize the treatment of children with cancer.

While measures for the assessment of the HRQOL are being developed for adults, in recent years, there have been both generic [20-22] and cancer specific [23,24] measures being developed in Japan. Additionally, there are very few measures for the assessment of the HRQOL for childhood cancer patients and survivors for which reliability and validity have been fully demonstrated [25-27], and there are none for survivors of specific cancers.

The Minneapolis-Manchester Quality of Life Survey of Health (MMQL) was designed as a comprehensive, multidimensional self-report instrument for measuring HRQOL among survivors of childhood cancer $[28,29]$, and is frequently utilized in other countries [30-35].

The present study aimed to develop a Japanese version of a globally comparable HRQOL measurement for childhood cancer survivors and investigate its reliability and validity. The development of the Japanese version of this measure for evaluation will serve to assist the construction of a long-term follow-up support system in Japan for childhood cancer survivors.

\section{Methods}

\section{Scale development}

We obtained permission from Dr. Smita Bhatia to translate the Minneapolis-Manchester Quality of Life Survey of Health Adolescent Form (MMQL-AF) into Japanese using a standardized validation procedure. Two translators, an American who had been living in Japan for many years and was fluent in Japanese and the other, a Japanese who was fluent in English independently translated the MMQL-AF into Japanese. The translators and the authors discussed the translation and whether it was conceptually equivalent to the original English version.
Then, a professional bilingual translator (English and Japanese) back translated the Japanese version of the MMQL-AF into English. There were only a few minor changes in the back translated Japanese version, and we conducted a pilot test on six children aged 13-18 years (mean age, $15.5 \pm 1.71$; 3 males, 3 females, 3 with cancer off therapy and 3 healthy controls). We asked children whether they understood the questions and noted the amount of time they needed to complete the questionnaire. There were no significant problems; therefore, we completed the Japanese version of MMQL-AF.

\section{Study population \\ Children with cancer}

We recruited children who met the following eligibility criteria (according to the original study of the MMQL) from eight hospitals in Japan.

Eligibility criteria were as follows: 1) Participants aged $\geq 13$ years and $<19$ years. The age category for the Minneapolis-Manchester Quality of Life Survey of Health Adolescent Form (MMQL-AF) is between 13 and 20 years of age, while the age category for the Japanese version of the Pediatric Quality of Life Inventory (PedsQL-J), which was used as a validation scale in the present study, is 13-18 years of age. Therefore, the upper age limit of the present study was determined to be $<19$ years of age; 2) Diagnosed with childhood cancer and off therapy for more than one year after completion of the treatment (in cases of recurrence, after completion of its treatment); 3) Informed consent for participation in the study was obtained from the parents/guardian as well as the children.

Exclusionary criteria were as follows: 1) Children diagnosed with cancer and still undergoing therapy; 2) Children whose mental and/or physical status did not allow for participation in the survey; 3) Children with a serious cognitive disorder; 4) Children with a serious chronic physical disease other than cancer; or 5) Any individuals whom the attending physician determined unsuitable for this study.

\section{Healthy controls}

We recruited children aged 13-18 years of age (with no history of cancer or any other chronic illness) from junior high school and high school in Tokyo.

\section{Procedure and measurement}

The children for whom informed consent for participation in this study had been obtained from the children themselves and the parents/guardians were asked to respond to the following questionnaires:

\section{Japanese version of Minneapolis-Manchester quality of life survey of health adolescent form (MMQL-AF)}

Minneapolis-Manchester Quality of Life Survey of Health (MMQL) is a self-report questionnaire recognized globally 
and allows for practical, comprehensive, and multidimensional measurement of HRQOL specific to childhood cancer survivors. Depending on the developmental stage of the children, two versions of the questionnaire, i.e., for children aged 8-12 years (MMQL-Youth Form) [29] and for those aged 13-20 years (MMQL-Adolescent Form) [28] have been developed at each language level. In the present study, the MMQL-AF was used. MMQL-AF consists of 46 items and is composed of the following seven subscales-physical functioning (9 items); cognitive functioning (9 items); psychological functioning (9 items); body image (6 items); social functioning (6 items); outlook on life (3 items); and human relationships (4 items).

\section{Japanese version of the pediatric quality of life inventory (PedsQL-J)}

The Japanese version of the HRQOL scale for children, which was developed by Kobayashi et al. [25], was used in the present study for children ages 13-18 years. It consists of 23 items and the following four subscales: physical functioning (8 items); emotional functioning (5 items); social functioning (5 items); and school functioning (5 items). Additionally, its reliability and validity have been previously demonstrated. This scale is used as a standard for the investigation of the validity of the Japanese version of MMQL-AF, with approval from Dr. James W. Varni, the author of the original version, MAPI Research TRUST, the copyright holding organization, and Dr. Kyoko Kobayashi and Dr. Kiyoko Kamibeppu, the authors of the Japanese version.

\section{Scale for the consciousness of self-affirmation}

The scale for the Consciousness of Self-affirmation is a scale developed by Hiraishi [36,37], which focuses on psychological health during adolescence. This scale is used to discuss the influence of self-esteem specific to adolescents using a developmental perspective in order to deepen the psychological understanding of childhood cancer survivors during adolescence. This scale consists of 41 items in a questionnaire format divided into two fields, i.e., an intrapersonal field and an interpersonal field. The lower-level components for each field are as follows: Lower-level components for the intrapersonal field include self-acceptance (4 items); attitude of self-actualization (7 items); and sense of fulfillment (8 items). The intrapersonal field is related to psychological health in adolescence, especially self-esteem/self-image. Lower-level components for the interpersonal field include the following scales: self-closure/misanthropy (8 items); self-expression/interpersonal positivity (7 items); and sense of being evaluated/ interpersonal tension (7 items). The interpersonal field is related to social competence, such as interpersonal tension, interpersonal distrust, or positive interpersonal attitude. This scale is also used as a standard for the investigation of the validity of the Japanese version of the MMQL-AF.

For healthy controls, a question assessing whether they had any chronic illness or disease that required longterm treatment in the hospital was added.

For children with cancer, information on the following items was obtained from the physician of the cooperating institution for the study: 1) Child's sex; 2) Child's age; 3) Name of the disease; 4) Date of diagnosis or date of start of treatment; 5) Date of completion of treatment; 6) History of recurrence (if yes, provide the date of the completion of treatment after recurrence); 7) History of surgery (If yes, provide the site of surgery); 8) History of chemotherapy; 9) History of radiotherapy (If yes, provide the site of irradiation and dosage); 10) History of hematopoietic stem cell transplantation (If yes, provide (a) type, (b) source of stem cell, (c) number of times); and 11) Experience of late effects.

\section{Conducting the survey}

At cooperating institutions for the study, approval by the Institutional Review Board was obtained after a review, and approval by the head of the institution was obtained.

\section{Children with cancer}

Physicians selected prospective subjects based on the eligibility criteria of this study, explained the purpose and contents to the parents/guardians both orally and in writing, and obtained their consent.

In the explanation document, the term "disease that requires long-term treatment in the hospital" was used instead of "childhood cancer" as there may be some cases where the "childhood cancer" disease was kept a secret from the child.

After obtaining informed consent for participation in this survey from the parents/guardians, the physician explained the purpose and the contents of this study to the children both orally and with written documents.

When children agreed to participate in this study, they completed the survey form, which was a self-report questionnaire, without providing their name, and mailed it in to the researcher (MK).

\section{Healthy controls}

Junior high school and high school children and their parents/guardians received an explanatory pamphlet and questionnaire package. When children and the parents/ guardians agreed to participate in the survey, children completed the questionnaire, without providing their name, and mailed it in to the researcher (MK). 


\section{Test-retest reliability}

Test-retest reliability was assessed at the National Cancer Center Hospital. Thirty children and their parents/ guardians agreed to take a retest after two weeks. The questionnaires were mailed to the families two weeks after the initial assessments and were all returned within two weeks.

\section{Statistical analysis}

Statistical analyses of the study were conducted using SPSS 20.0 J for Windows (SPSS, Inc., Chicago, IL) and the significance level was set at $p<0.05$.

Factor analysis was used to confirm the structure of the subscales of the Japanese version of the MMQL-AF. Reliability was determined by internal consistency using Cronbach's alpha and test-retest reliability using Spearman's intra-class correlation coefficient (ICC). Internal consistency was considered good when Cronbach's alpha exceeded 0.70 [38].

Concurrent validity was assessed by Spearman's correlation coefficient between the MMQL-AF and PedsQL-J. Discriminate validity was determined by comparing the participant groups (children with cancer and healthy controls).

Feasibility was determined by the percentage of missing values and by the amount of time required to complete the questionnaires.

\section{Ethical considerations}

The present study was approved by the Institutional Review Board at each participating institution and Tokyo Medical Dental University.

\section{Results}

\section{Subject characteristics}

The present study included 141 children with cancer who completed treatment at least one year prior and 183 healthy controls with no previous history of cancer or other chronic diseases. Table 1 presents the characteristics of the study population. The mean time after completion of treatment was 6.23 years (ranging from 1.0 to 15.25 years) for the children with cancer group. There were no significant differences between the groups with regard to age $(t(322)=0.12, p=0.91$, ns $)$ and sex $\left(\chi^{2}(1)=4.08, p=0.13, \mathrm{~ns}\right)$. A total of 88 subjects $(62.4 \%)$ in the children with cancer group had hematological diseases, while the remaining subjects had solid tumors.

\section{Structure of the MMQL-AF}

To confirm the scale structure of the Japanese version of the MMQL-AF, factor analysis (principal factor analysis) was conducted using the 46 items. In this analysis, a factor loading value $\geq 0.40$ was considered sufficiently high. Based on changes in Eigenvalues and the interpretability
Table 1 Descriptive statistics of the study population

\begin{tabular}{|c|c|c|c|c|}
\hline & \multicolumn{2}{|c|}{$\begin{array}{l}\text { Children with } \\
\text { cancer off therapy } \\
(n=141)\end{array}$} & \multicolumn{2}{|c|}{$\begin{array}{l}\text { Healthy } \\
\text { controls } \\
(n=183)\end{array}$} \\
\hline & $\bar{n}$ & $\%$ & $\bar{n}$ & $\%$ \\
\hline \multicolumn{5}{|l|}{ Age, years } \\
\hline Mean (SD) & \multicolumn{2}{|c|}{$15.75(1.79)$} & \multicolumn{2}{|c|}{$15.73(2.03$} \\
\hline \multicolumn{5}{|l|}{ Sex } \\
\hline Male & 81 & 57.4 & 86 & 47.0 \\
\hline Female & 60 & 42.6 & 96 & 52.5 \\
\hline Unknown & & & 1 & .5 \\
\hline \multicolumn{5}{|l|}{ Diagnosis } \\
\hline Acute lymphoblastic leukemia & 46 & 32.6 & & - \\
\hline Acute myeloid leukemia & 11 & 7.8 & & - \\
\hline Other leukemia & 8 & 5.7 & & - \\
\hline Non-Hodgkin lymphoma & 23 & 16.3 & & - \\
\hline Osteosarcoma & 15 & 10.6 & & - \\
\hline Neuroblastoma & 6 & 4.3 & & - \\
\hline Rhabdomyosarcoma & 5 & 3.5 & & - \\
\hline Brain tumor & 5 & 3.5 & & - \\
\hline Hepatoblastoma & 3 & 2.1 & & - \\
\hline Wilms tumor & 1 & .8 & & - \\
\hline Other cancer & 18 & 12.8 & & - \\
\hline
\end{tabular}

of factors, it appeared that a 6-factor structure was appropriate rather than the 7-factor structure of the original version. After excluding two items with no sufficient factor loadings ("Feeling inferior to most people" and "Prefer to watch rather than take part in games and sports"), factor analysis was again conducted with the remaining 44 items on a hypothesized 6-factor structure. Table 2 presents the final factor pattern and inter-factor correlations after using a Promax rotation. The social functioning and intimate relations factors in the original version were combined into a single factor. It should be noted that the cumulative contribution ratio before rotation was $56.83 \%$.

\section{Reliability}

To assess the internal consistency of the Japanese version of the MMQL-AF, Cronbach's alpha coefficient was calculated. Results showed sufficient Cronbach's alpha coefficients for factors one to six ( 0.83 to 0.89 , respectively) (see Table 2).

Table 3 presents the test-retest reliability analysis of the Japanese version of the MMQL-AF. Excellent ICC values $>0.90$ were obtained with the exception of psychological functioning, for which the ICC value was good to fair at 0.79 . 
Table 2 Factor analysis of the Japanese version of the MMQL-AF

\begin{tabular}{llllll} 
Factor 1 & Factor 2 & Factor 3 & Factor 4 & Factor 5 & Factor 6 \\
\hline
\end{tabular}

Factor 1: Social functioning \& intimate relations

Have many close friends

Getting along well with people their age

$\begin{array}{llllll}\mathbf{. 8 9} & .00 & .00 & -.09 & -.05 & .03 \\ \mathbf{. 8 5} & -.01 & -.01 & .04 & -.09 & -.04 \\ \mathbf{. 7 5} & .04 & -.12 & -.09 & -.09 & .05 \\ . \mathbf{7 5} & -.09 & -.04 & -.03 & -.03 & .11 \\ \mathbf{. 7 3} & -.07 & .00 & -.07 & .08 & .02 \\ \mathbf{- . 6 3} & -.17 & -.04 & -.05 & -.06 & .06 \\ \mathbf{- . 5 7} & -.07 & -.08 & -.16 & -.11 & .13 \\ \mathbf{. 5 3} & .04 & .05 & .04 & .20 & -.03 \\ \mathbf{. 4 9} & -.08 & .15 & .06 & -.11 & .11 \\ \mathbf{. 4 8} & -.05 & .00 & .07 & .10 & -.03\end{array}$

Having a lot in common with their friends

Being together with other people gives them a good feeling

Having similar hobbies and interests to people their age

Feel left out in groups of people their age

Difficulty in making friends

Find it easy to have an intimate relationship

Believing that people like to be with them

Feel confident when they are with people of opposite sex

\section{Factor 2: Psychological functioning}

$-.01$

Feeling sad

Feeling nervous or anxious

Feeling frightened

Feeling lonely

\section{$-.01$}

.01

$-.07$

Feeling angry

Worried about things in general

Worried about dying

Feeling tired during the day

Worried about their health

\section{Factor 3: Cognitive functioning}

Difficulty with school work compared with others in class

Homework or study is hard for them

Difficulty in concentrating at school

Difficulty in concentrating at work or school

Needing more help with school work than others in class

Difficulty with math or calculations

Difficulty with reading or writing

Difficulty with remembering things at school/college

Difficulty concentrating at other times (computer/games/playing cards/reading)

\section{Factor 4: Physical functioning}

Unable to keep up with others of their age when taking part in sports

Unable to do many activities because of health

Unable to do many activities because of arms or legs

Have a lot of energy for running or sports

Feel strong and healthy

Need time during day to rest

Have a lot of energy

Factor 5: Body image

Liking their body the way it is

Feelings about their body development

Being happy about the way they look

Feeling uncomfortable about the way their body is developing

.13

$-.18$

$\begin{array}{lll}.88 & .03 \quad-.03\end{array}$

$-.08$

.01

.88

.05

$-.07$

.02

$-.07$

.87

.09

$-.04$

$-.01$

$-.01$

$\begin{array}{ll}.80 & .05\end{array}$

$-.10$

.01

$-.12$

$\begin{array}{lll}.05 & .73 & .01\end{array}$

.02

$-.11$

.04

$.00 \quad .60$

$-.08 \quad .50$

$-.15$

$-.02$

.11

.09

$.03 \quad .49$

$.49 \quad-.04$

$-.13$

.06

.06

.41

$-.12$

.34

.02

.03

$\begin{array}{llllll}-.03 & .05 & .80 & -.10 & .01 & -.03 \\ .06 & .03 & -.77 & -.11 & .06 & -.06 \\ .07 & .01 & -.70 & -.12 & .06 & -.08 \\ -.01 & -.06 & .69 & -.03 & .20 & .00 \\ .00 & .06 & -.66 & -.07 & .06 & -.14 \\ .11 & -.08 & .64 & -.09 & .16 & -.09 \\ -.03 & .12 & .62 & -.04 & -.03 & -.13 \\ .05 & .07 & .61 & .02 & -.03 & .05 \\ -.05 & -.03 & -.43 & .03 & .08 & -.14\end{array}$

\begin{tabular}{llllll}
-.03 & .05 & .80 & -.10 & .01 & -.03 \\
.06 & .03 & -.77 & -.11 & .06 & -.06 \\
.07 & .01 & -.70 & -.12 & .06 & -.08 \\
-.01 & -.06 & .69 & -.03 & .20 & .00 \\
.00 & .06 & -.66 & -.07 & .06 & -.14 \\
.11 & -.08 & .64 & -.09 & .16 & -.09 \\
-.03 & .12 & .62 & -.04 & -.03 & -.13 \\
.05 & .07 & .61 & .02 & -.03 & .05 \\
-.05 & -.03 & -.43 & .03 & .08 & -.14 \\
\hline & & & & &
\end{tabular}

.15

$\begin{array}{ll}.18 & .14\end{array}$

$\begin{array}{ll}-.05 & -.03\end{array}$

$\begin{array}{lll}-.05 & -.14 & .03 \\ .13 & .02 & .01 \\ -.04 & .06 & .01 \\ .18 & -.06 & -.03 \\ .04 & -.01 & -.01 \\ .00 & -.11 & -.24 \\ .15 & .16 & -.16\end{array}$

$.03-80$

$\begin{array}{llllll}-.08 & -.02 & -.02 & .02 & .74 & .15 \\ .01 & .06 & -.06 & .01 & .72 & .03 \\ .11 & -.02 & .04 & -.24 & .68 & .11 \\ .01 & .05 & .01 & -.26 & -.68 & .07\end{array}$


Table 2 Factor analysis of the Japanese version of the MMQL-AF (Continued)

\begin{tabular}{|c|c|c|c|c|c|c|}
\hline Being satisfied about their weight & -.04 & .05 & .00 & -.19 & .66 & .00 \\
\hline Feeling that others think that their body is poorly developed & -.03 & -.04 & -.09 & -.16 & -.59 & .18 \\
\hline \multicolumn{7}{|l|}{ Factor 6: Outlook on life } \\
\hline Satisfied with the current life situation & .02 & .01 & .04 & -.02 & -.04 & .83 \\
\hline Happy with life in general & -.03 & .06 & .06 & .00 & .08 & .77 \\
\hline Happy with the way things are & .10 & .01 & .03 & -.12 & .07 & .65 \\
\hline Factor correlation & Factor 1 & Factor 2 & Factor 3 & Factor 4 & Factor 5 & Factor 6 \\
\hline Factor 1 & - & .56 & .47 & .40 & .43 & .42 \\
\hline Factor 2 & & - & .44 & .37 & .47 & .50 \\
\hline Factor 3 & & & - & .36 & .36 & .37 \\
\hline Factor 4 & & & & - & .24 & .34 \\
\hline Factor 5 & & & & & - & .43 \\
\hline Factor 6 & & & & & & - \\
\hline a & .89 & .89 & .88 & .83 & .83 & .84 \\
\hline
\end{tabular}

Extraction method is principle factor analysis using Promax rotation with Kaiser normalization.

Factor loadings greater than 0.40 are shown in boldface.

MMQL-AF: Minneapolis-Manchester Quality of Life Survey of Health - Adolescent Form., a: Cronbach's coefficient.

\section{Validity}

To assess the concurrent validity of the Japanese version of the MMQL-AF, Spearman's correlation coefficients were calculated between subscale scores for the Japanese version of the MMQL-AF and the PedsQL-J (Table 4). The physical functioning scale on the MMQL-AF was strongly correlated with the physical functioning scale and weakly correlated with each of the other scales on the PedsQL-J. Each of the psychological, social functioning and intimate relations, and outlook on life scales of the MMQL-AF was moderately correlated with each of the physical, emotional, and social functioning scales and weakly correlated with the school functioning scale of the PedsQL-J. The body image scale on the MMQL-AF was weakly correlated with all scales on the PedsQL-J. The cognitive functioning scale of the MMQL-AF was moderately correlated with all scales on the PedsQL-J.

Table 3 Test-retest reliability of the Japanese version of the MMQL-AF $(n=30)$

\begin{tabular}{llllllllll}
\hline & \multicolumn{1}{l}{ First } & & & \multicolumn{2}{l}{ Second } & & ICC \\
\cline { 2 - 3 } MMQL-AF questionnaire & Mean & SD & & Mean & SD & \\
\hline Physical functioning & 15.41 & 6.01 & 14.90 & 6.07 & $.96^{* *}$ \\
Psychological functioning & 19.17 & 6.83 & 17.97 & 5.70 & $.79^{* *}$ \\
Body image & 15.33 & 4.39 & 15.20 & 4.45 & $.90^{* *}$ \\
Social functioning \& intimate relations & 19.86 & 6.39 & 20.28 & 7.33 & $.90^{* *}$ \\
Cognitive functioning & 15.45 & 5.96 & 15.21 & 6.22 & $.92^{* *}$ \\
Outlook on life & 6.30 & 2.98 & 5.97 & 3.07 & $.90^{* *}$ \\
\hline
\end{tabular}

MMQL-AF: Minneapolis-Manchester Quality of Life Survey of Health-Adolescent Form, n: number of individuals, SD: standard deviation, ICC: intraclass correlation coefficient. ${ }^{* *} p<0.01$.
To assess the discriminant validity of the Japanese version of the MMQL-AF, scores for each subscale on the MMQL-AF were compared between children with cancer and healthy controls (Table 5). Results indicated that the children with cancer had significantly $(<0.01)$ higher scores for the physical functioning scale compared to the healthy controls. Compared with the healthy controls, the physical functioning scale on the PedsQL-J, which was strongly correlated with the physical functioning scale on the MMQL-AF (Table 4), also showed significantly $(<0.01)$ higher scores among children with cancer (Table 6). Comparisons of the scores for each subscale on the MMQL-AF between the children with cancer and the healthy controls demonstrated that children with cancer showed significantly $(<0.05)$ higher scores on the social functioning and intimate relations and cognitive functioning scales compared to the healthy controls (Table 5). For the consciousness of self-affirmation scale, significant differences in the scores were found between children with cancer and the healthy controls on both the interpersonal and intrapersonal fields $(<0.05$, Table 7$)$.

\section{Feasibility}

The percentage of missing values in the present study was $0.40 \%$. During pilot testing, all the items on the MMQL-AF were completed in 6-12 minutes.

\section{Discussion}

In Japan, there are estimated to be over several million childhood cancer survivors, and the number of childhood cancer survivors is reported to be increasing at a rate of $\geq 1400$ individuals per year [39]. In addition to 
Table 4 Spearman's correlation coefficients between the Japanese version of the MMQL-AF and the PedsQL-J $(n=324)$

\begin{tabular}{lllll}
\hline \multirow{2}{*}{ MMQL-AF questionnaire } & \multicolumn{2}{l}{ PedsQL-J } & & \\
\cline { 2 - 5 } & Physical functioning & Emotional functioning & Social functioning & School functioning \\
\hline Physical functioning & $.720^{* *}$ & $.345^{* *}$ & $.361^{* *}$ & $.353^{* *}$ \\
Psychological functioning & $.454^{* *}$ & $.593^{* *}$ & $.527^{* *}$ & $.359^{* *}$ \\
Body image & $.381^{* *}$ & $.390^{* *}$ & $.385^{* *}$ & $.307^{* *}$ \\
Social functioning \& intimate relations & $.437^{* *}$ & $.460^{* *}$ & $.649^{* *}$ & $.286^{* *}$ \\
Cognitive functioning & $.516^{* *}$ & $.413^{* *}$ & $.462^{* *}$ & $.460^{* *}$ \\
Outlook on life & $.424^{* *}$ & $.472^{* *}$ & $.424^{* *}$ & $.366^{* *}$ \\
\hline
\end{tabular}

MMQL-AF: Minneapolis-Manchester Quality of Life Survey of Health-Adolescent Form, PedsQL-J: the Japanese version of the Pediatric Quality of Life Inventory, $\mathrm{n}$ : number of individuals. ${ }^{* *} p<0.01$.

complications with intensive treatment, childhood cancer is associated with problems following treatment during the growth and development period, such as failure to thrive/developmental disorders, endocrine disorders, organ damage, gonadal disturbances, high brain dysfunction, and second primary cancers, even after a cure is achieved. To provide long-term support for childhood cancer survivors in their daily lives and school attendance/employment after completion of treatment, long-term follow-up should involve HRQOL assessments and appropriate measures from a multidimensional perspective. These should include not only the medical-physical aspects, but also the psychosocial aspects according to the developmental process. Although both generic and disease-specific multidimensional measures for children and adolescents have been developed in Western countries, only limited HRQOL measures for children and adolescents with established reliability and validity are available in Japan [25-27].

The Pediatric Quality of Life Inventory (PedsQL) is a modular instrument designed to measure HRQOL in children and adolescents aged $2-18$ years. The Japanese version of the PedsQL 4.0 Generic Core Scales was standardized by Kobayashi et al. [25] and was used to assess the concurrent validity in the present study. In 2011, the Japanese version of the PedsQL 3.0 Cancer Modular was standardized by Tsuji et al. [27]. The PedsQL 3.0 Cancer Modular is designed to assess a wide age range of 2-18 years with proxy assessments by guardians used for younger children. It is a measure designed for children with cancer, although the standardization of the Japanese version included individuals who had completed treatment at least a year prior.

The Japanese version of the MMQL-AF is a selfadministered HRQOL instrument specific to childhood cancer survivors aged 13-18 years (adolescents falling under the categories of junior high and high school students in Japan) who have completed treatment.

Factor analysis of the Japanese version of the MMQL-AF showed different results from those obtained for the original version of the MMQL-AF [28]. First, two items with non-sufficient factor loadings were excluded. Second, the social functioning and intimate relations scales in the original version were combined into a single factor. Although the intimate relations scale was originally composed of four items ("Difficulty in making friends," "Feel left out in groups of people their age," "Feel confident when they are with people of the opposite sex," and "Find it easy to have an intimate relationship") [28], the subsequent final version of the MMQL-AF has "Difficulty in making friends" and "Feel left out in groups of people their age" included in the social functioning subscale and "Feel confident when they are with people of the opposite sex" and "Find it easy to have an intimate relationship" included in the intimate relations subscale. Considering that the instructions

Table 5 Discriminant validity involving known group analysis for the Japanese version of the MMQL-AF

\begin{tabular}{|c|c|c|c|c|c|c|}
\hline \multirow[b]{2}{*}{ MMQL-AF questionnaire } & \multicolumn{2}{|c|}{ Children with cancer off therapy $(n=141)$} & \multicolumn{2}{|c|}{ Healthy controls $(n=183)$} & \multirow[t]{2}{*}{$t$} & \multirow[t]{2}{*}{$p$ value } \\
\hline & Mean & SD & Mean & SD & & \\
\hline Physical functioning & 13.62 & 5.36 & 8.92 & 2.47 & 9.46 & $<0.01$ \\
\hline Psychological functioning & 20.32 & 7.87 & 19.59 & 7.48 & .84 & ns \\
\hline Body image & 15.30 & 4.56 & 14.98 & 3.98 & .67 & ns \\
\hline Social functioning \& intimate relations & 20.37 & 7.76 & 18.67 & 5.96 & 2.13 & $<0.05$ \\
\hline Cognitive functioning & 15.47 & 5.73 & 13.95 & 5.16 & 2.48 & $<0.05$ \\
\hline Outlook on life & 6.15 & 2.81 & 5.93 & 2.48 & .74 & ns \\
\hline
\end{tabular}

MMQL-AF: Minneapolis-Manchester Quality of Life Survey of Health-Adolescent Form, n: number of individuals, SD: standard deviation, $t: t$ value. 
Table 6 Discriminant validity involving known group analysis for the PedsQL-J

\begin{tabular}{|c|c|c|c|c|c|c|}
\hline \multirow[b]{2}{*}{ PedsQL-J } & \multicolumn{2}{|c|}{ Children with cancer off therapy $(n=141)$} & \multicolumn{2}{|c|}{ Healthy controls $(n=181)$} & \multirow[t]{2}{*}{$t$} & \multirow[t]{2}{*}{$p$ value } \\
\hline & Mean & SD & Mean & SD & & \\
\hline Physical functioning & 5.15 & 5.17 & 2.22 & 3.76 & 5.66 & $<0.01$ \\
\hline Emotional functioning & 5.35 & 4.06 & 5.27 & 3.61 & .18 & ns \\
\hline Social functioning & 2.85 & 3.50 & 2.52 & 2.80 & .92 & ns \\
\hline School functioning & 4.45 & 3.10 & 3.89 & 2.71 & 1.69 & ns \\
\hline
\end{tabular}

PedsQL-J: the Japanese version of the Pediatric Quality of Life Inventory, n: number of individuals, SD: standard deviation, $t: t$ value.

appearing on the intimate relations subscale on the questionnaire state "about close relationships with other people," combining this subscale with the social functioning factor, which asks about "the relationship people have with their family," is acceptable and rather natural for responders.

Assessment of the reliability of the Japanese version of the MMQL-AF found that all factors had Cronbach's coefficient alpha values $>0.80$, indicating evidence of high internal consistency.

For test-retest reliability, excellent ICC values $>0.90$ were obtained with the exception of psychological functioning, for which the ICC value was good to fair at 0.79 . Based on these results, it can be considered that the Japanese version of the MMQL-AF is a highly reliable measure.

For validation of the Japanese version of the MMQL-AF, Spearman's correlation coefficients were calculated between subscale scores for the Japanese version of the MMQL-AF and the PedsQL-J to assess the concurrent validity of the Japanese version of the MMQL-AF. Scores for all subscales in the MMQL-AF were significantly correlated with the scores for all subscales on the PedsQL-J. In particular, the physical functioning subscale on the Japanese version of the MMQL-AF was very strongly correlated with the physical functioning subscale on the PedsQL-J, and each of the psychological functioning, social functioning and intimate relations, and outlook on life scales on the MMQL-AF was moderately correlated with each of the physical, emotional, and social functioning scales of the PedsQL-J. The body image scale of the MMQL-AF was only weakly correlated with any scale of the PedsQL-J. Based on these results, the Japanese version of the MMQL-AF can be considered a structurally valid HRQOL instrument. The body image subscale can be considered a subscale characteristic of the MMQL-AF.

To assess the discriminant validity of the Japanese version of the MMQL-AF, scores for each subscale on the MMQL-AF were compared between children with cancer and healthy controls. Results indicated that children with cancer had significantly higher scores for the physical functioning subscale compared to the healthy controls, indicating that children with cancer tended to feel limitations during daytime activities. Compared to healthy controls, the physical functioning subscale on the PedsQL-J, which was strongly correlated with the physical functioning subscale on the MMQL-AF, also showed significantly $(<0.01)$ higher scores among the children with cancer, indicating that the physical functioning subscale on the MMQL-AF represented a measure of physical function in children with cancer.

Comparisons of the scores for each subscale on the MMQL-AF between the children with cancer and the healthy controls demonstrated that children with cancer showed significantly higher scores for the social functioning and intimate relations subscales compared to healthy controls, indicating that children with cancer tended to have greater anxiety about relations with others. The children with cancer also showed significantly higher scores for the cognitive functioning subscale compared with the healthy controls, indicating that children with cancer tended to have a greater feeling of lack of fulfillment in school or work. The scale for the consciousness of selfaffirmation is a measure related to psychological health during adolescence, which consists of the intrapersonal field containing the three components of self-acceptance, attitude of self-actualization, and sense of fulfillment and the interpersonal field containing the three components of self-closure/misanthropy, self-expression/positive

Table 7 Discriminant validity involving known group analysis of the scale for the consciousness of self-affirmation

\begin{tabular}{|c|c|c|c|c|c|c|}
\hline \multirow[b]{2}{*}{ Scale for the consciousness of self-affirmation } & \multicolumn{2}{|c|}{ Children with cancer off therapy $(n=140)$} & \multicolumn{2}{|c|}{ Healthy controls $(n=181)$} & \multirow[t]{2}{*}{$t$} & \multirow[t]{2}{*}{$p$ value } \\
\hline & Mean & SD & Mean & SD & & \\
\hline Intrapersonal field ${ }^{\mathrm{a}}$ & 46.85 & 7.32 & 44.87 & 9.13 & 2.15 & $<0.05$ \\
\hline Interpersonal field ${ }^{b}$ & 72.05 & 10.59 & 74.35 & 9.83 & 1.98 & $<0.05$ \\
\hline
\end{tabular}

$\mathrm{n}$ : number of individuals, SD: standard deviation, $t: t$ value.

${ }^{a}$ self-acceptance, attitude of self-actualization, sense of fulfillment.

${ }^{b}$ self-closure/misanthropy, self-expression/interpersonal positivity, sense of being evaluated/interpersonal tension. 
interpersonal attitude, and sense of being evaluated/interpersonal tension.

No differences in any component were found between children with cancer and healthy controls, although there were significant differences between children with cancer and the healthy controls with regard to scores for both the intrapersonal field and the interpersonal field. The intrapersonal field was related to psychological health during adolescence, especially self-esteem/self-image, and higher scores for this field indicate lower self-esteem/selfimage. In contrast, the interpersonal field was related to social competence, such as interpersonal tension, interpersonal distrust, or positive interpersonal attitude and lower scores for this field indicate lower social competence. Comparisons of scores for each subscale on the MMQLAF between the children with cancer and the healthy controls showed a significantly higher score for the social functioning and intimate relations subscales among children with cancer compared to the healthy controls. Additionally, the observed tendency for children with cancer to have greater anxiety about relations with others may be explained by lower social competence among them compared to healthy controls as evidenced by the interpersonal field of the scale for the consciousness of self-affirmation. In addition, a significantly higher score for the cognitive functioning subscale on the MMQL-AF was obtained among the children with cancer compared with the healthy controls, and the observed tendency for children with cancer to have a greater feeling of lack of fulfillment in school or work may be explained by a tendency towards lower self-esteem/self-image among children with cancer compared to the healthy controls as evidenced in the intrapersonal field of the scale for the consciousness of self-affirmation. There were no significant differences between children with cancer and healthy controls with regard to the scores for any of the psychological functioning, body image, and outlook on life subscales.

The percentage of missing values in the present study was $0.40 \%$. During pilot testing, all the items on the MMQL-AF were completed in 6-12 minutes, indicating acceptable feasibility of the Japanese version of the MMQL-AF.

\section{Limitations and future direction}

The main purpose of the present study was to examine the validity and reliability of the Japanese version of the MMQL-AF; however, the present study did not examine the scale's responsiveness to change. Responsiveness to change is an important factor for predicting how and why children with cancer will change over time. Thus, further examination of the measure and its responsiveness to change is warranted in future studies.
As the number of participants with each cancer type was limited in this study, future researchers may want to conduct more elaborate procedures in order to determine the effects and impact of each cancer type within the recovery process. The present study did find significant differences between the hematological disease group and the solid tumor group, as well as between the male and female groups. Children in the solid tumor group tended to have restricted mobility in physical functioning $(\mathrm{t}(140)=-3.06, p<0.01)$, and males tended to display negative emotions in psychological functioning $(\mathrm{t}(140)=-2.80, p<0.01)$.

\section{Conclusion}

The results of the present study showed evidence for the reliability and validity of the MMQL-AF as a comprehensive, multidimensional self-report instrument for measuring HQOL among adolescent survivors of childhood cancer. As Dr. Smita Bhatia mentioned in the original study, the instrument has been tested in a random sample of healthy children who had no chronic illness, and thus provides normative data that can be used in future studies for comparing HRQOL in various populations.

\section{Abbreviations \\ HRQOL: Health-related quality of life; ICC: Intra-class correlation coefficient; MMQL: Minneapolis-Manchester quality of life survey of health; MMQL-AF: Minneapolis-Manchester quality of life survey of health adolescent form; PedsQL: Pediatric quality of life inventory; PedsQL-J: Japanese version of PedsQL; QOL: Quality of life; SD: Standard deviation; SPSS: Statistical package for social science.}

\section{Competing interests}

The authors declare that they have no competing interests.

\section{Authors' contributions}

MK and EM conceptualized the rationale and design of the study. $\mathrm{HH}$, TR, $\mathrm{AH}, \mathrm{NT}, \mathrm{TY}$ and HU coordinated participants and settings in each hospital. After approval of each Institutional Review Board, they administered questionnaires to children with cancer and their parents/guardians and collected data. MK and EM conducted statistical analyses and drafted the manuscript. All authors read and approved the final version of the manuscript.

\section{Acknowledgements}

We would like to deeply thank all children and their parents/guardians who willingly cooperated with this research. We also gratefully acknowledge Yasushi Ishida M.D., Ph.D. for significant support and advice. We are grateful to Dr. Smita Bhatia for permission to develop the Japanese-language version of the MMQL.

The authors would also like to thank the following participating institutions:

1. National Cancer Center Hospital

2. Mie University Hospital

3. Tohoku University Hospital

4. Kobe University Hospital

5. Tokyo Metropolitan Children's Medical Center

6. St. Luke's International Hospital

7. Chiba Cancer Center

8. National Center for Global Health and Medicine Center Hospital

9. Mejiro Kenshin Junior and Senior High School 


\section{Author details}

'Section of Liaison Psychiatry and Palliative Medicine, Graduate School of Tokyo Medical and Dental University, 1-5-45 Yushima, Bunkyo-ku, Tokyo 113-8519, Japan. ${ }^{2}$ Graduate School of Psychology, Mejiro University, 4-31-1 Nakaochiai, Shinjuku-ku, Tokyo 161-8539, Japan. ${ }^{3}$ Department of Medical Education/Pediatrics, Mie University Graduate School of Medicine, 2-174 Edobashi, Tsu-shi, Mie 514-8507, Japan. ${ }^{4}$ Department of Pediatrics, Tohoku University School of Medicine, 1-1 Seiryou-machi, Aoba-ku, Sendai-shi, Miyag 980-8574, Japan. ${ }^{5}$ Department of Pediatrics, Kobe University Graduate School of Medicine, 7-5-1 Kusunokicho, Chuo-ku, Kobe-shi, Hyogo 650-0017, Japan. ${ }^{6}$ Niizashiki Central General Hospital, Department of Palliative Medicine, 1-7-2 Tohoku, Niiza-shi, Saitama 352-0001, Japan. 'Division of Orthopedic Surgery, Chiba Cancer Center, 666-2 Nitona-cho, Chuo-ku, Chiba 260-8717, Japan.

${ }^{8}$ National Center for Global Health and Medicine, Department of Pediatrics, 1-21-1 Toyama, Shinjuku-ku, Tokyo 162-8655, Japan.

Received: 18 November 2013 Accepted: 7 August 2014

Published: 15 August 2014

\section{References}

1. Schwartz CL, Hobbie WL, Constine LS, Ruccione KS: Survivors of Childhood and Adolescent Cancer. Berlin: Springer-Verlag; 2005.

2. Jemal A, Siegel R, Ward E, Hao Y, Xu J, Thun MJ: Cancer statistics, 2009. CA Cancer J Clin 2009, 59:225-249.

3. Pizzo AP, Poplack DG: Principles and Practice of Pediatric Oncology. Philadelphia: Lippincott Williams \& Wilkins: 2012.

4. Oeffinger KC, Mertens AC, Sklar CA, Kawashima T, Hudson MM, Meadows AT, Friedman DL, Marina N, Hobbie W, Kadan-Lottick NS, Schwartz CL, Leisenring W, Robison LL: Chronic health conditions in adult survivors of childhood cancer. N Engl J Med 2006, 355:1572-1582.

5. Schwartz $\mathrm{CL}$ : Long-term survivors of childhood cancer: the late effects of therapy. Oncologist 1999, 4:45-54

6. Smith M, Hare ML: An Overview of progress in childhood cancer survival. J Pediatr Oncol Nurs 2004, 21:160-164.

7. Robinson LL, Bhatia S: Late-effects among survivors of leukemia and lymphoma during childhood and adolescence. Br J Haematol 2003, 122:345-359.

8. Canadian Cancer Society/National Cancer Institute of Canada: Canadian Cancer Statistics 2008. Toronto, ON: Canadian Cancer Society/National Cancer Institute of Canada; 2008.

9. Boman KK: Assessing psychological and health-related quality of life (HRQL) late effects after childhood cancer. Acta Paediatr 2007, 96:1265-1268.

10. Pemberger S, Jagsch R, Frey E, Felder-Puig R, Gadner H, Kryspin-Exner I, Topf R: Quality of life in long-term childhood cancer survivors and the relation of late effects and subjective well-being. Support Care Cancer 2005, 13:49-56.

11. Stam H, Grootenhuis M, Last B: The course of life of survivors of childhood cancer. Psychooncology 2005, 14:227-238.

12. Erikson EH: Identity and the Life Cycle. New York: International Universities Press; 1959.

13. Stanwyck DJ: Self-esteem through the life span. Fam Community Health 1983, 6:11-28.

14. Evans SE, Radford M: Current lifestyle of young adults treated for cancer in childhood. Arch Dis Child 1995, 72:423-426.

15. Langeveld NE, Stam H, Grootenhuis MA, Last BF: Quality of life in young adult survivors of childhood cancer. Support Care Cancer 2002, 10:579-600.

16. Langeveld NE, Grootenhuis MA, Voûte PA, de Haan RJ, van den Bos C: Quality of life, self-esteem and worries in young adult survivors of childhood cancer. Psychooncology 2004, 13:867-881

17. Eiser C: Beyond survival: quality of life and follow-up after childhood cancer. J Pediatr Psychol 2007, 32:1140-1150.

18. Ishida Y, Honda M, Ozono S, Okamura J, Asami K, Maeda N, Sakamoto N, Inada H, Iwai T, Kamibeppu K, Kakee N, Horibe K: Late effects and quality of life of childhood cancer survivors: part 1. Impact of stem cell transplantation. Int J Hematol 2010, 91:865-876.

19. Ishida Y, Sakamoto N, Kamibeppu K, Kakee N, Iwai T, Ozono S, Maeda N, Okamura J, Asami K, Inada H, Honda M, Horibe K: Late effects and quality of life of childhood cancer survivors: part 2. Impact of radiotherapy. Int J Hematol 2010, 92:95-104.

20. Fukuhara S, Bito S, Green J, Hsiao A, Kurokawa K: Translation, adaptation, and validation of the SF-36 Health Survey for use in Japan. J Clin Epidemiol 1998, 51:1037-1044.

21. Fukuhara S, Ware JE, Kosinski M, Wada S, Gandek B: Psychometric and clinical tests of validity of the Japanese SF-36 Health Survey. J Clin Epidemiol 1998, 51:1045-1053.

22. Tsuchiya A, Ikeda S, Ikegami N, Nishimura S, Sakai I, Fukuda T, Hamashima C, Hisashige A, Tamura M: Estimating an EQ-5D population value set: the case of Japan. Health Econ 2002, 11:341-353.

23. Kobayashi K, Takeda F, Teramukai S, Gotoh I, Sakai H, Yoneda S, Noguchi Y, Ogasawara H, Yoshida K: A cross-validation of the European Organization for Research and Treatment of Cancer QLQ-C30 (EORTC QLQ-C30) for Japanese with lung cancer. Eur J Cancer 1998, 34:810-815.

24. Fumimoto $\mathrm{H}$, Kobayashi $\mathrm{K}$, Chang $\mathrm{CH}$, Eremenco $\mathrm{S}$, Fujiki $\mathrm{Y}$, Uemura $\mathrm{S}$, Ohashi Y, Kudoh S: Cross-cultural validation of an international questionnaire, the general measure of the Functional Assessment of Cancer Therapy scale (FACT-G), for Japanese. Qual Life Res 2001, 10:701-709.

25. Kobayashi K, Kamibeppu K: Measuring quality of life in Japanese children: development of the Japanese version of PedsQL. Pediatr Int 2010, 52:80-88.

26. Sato I, Higuchi A, Yanagisawa T, Mukasa A, Ida K, Sawamura Y, Sugiyama K, Saito N, Kumabe T, Terasaki M, Nishikawa R, Ishida Y, Kamibeppu K: Development of the Japanese version of the pediatric quality of life inventory brain tumor module. Health Qual Life Outcomes 2010, 8:38.

27. Tsuji N, Kakee N, Ishida Y, Asami K, Tabuchi K, Nakadate H, Iwai T, Maeda M, Okamura J, Kazama T, Terao Y, Ohyama W, Yuza Y, Kaneko T, Manabe A, Kobayashi K, Kamibeppu K, Matsushima E: Validation of the Japanese version of the Pediatric Quality of Life Inventory (PedsQL) cancer module. Health Qual Life Outcomes 2011, 9:22.

28. Bhatia S, Jenny ME, Bogue MK, Rockwood TH, Feusner JH, Friedman DL, Robison LL, Kane DL: The Minneapolis-Manchester quality of life instrument: reliability and validity of the adolescent form. J Clin Oncol 2002, 20:4692-4698.

29. Bhatia S, Jenny ME, Wu E, Bogue MK, Rockwood TH, Feusner JH, Friedman $\mathrm{DL}$, Robison LL, Kane RL: The Minneapolis-Manchester quality of life instrument: reliability and validity of the youth form. J Pediatr 2004, 145:39-46.

30. Shankar S, Robinson L, Jenny ME, Rockwood TH, Wu E, Feusner J, Friedman $D$, Kane RL, Bhatia S: Health-related quality of life in young survivor of childhood cancer using the Minneapolis-Manchester quality of life-youth form. Pediatrics 2005, 115:435-442.

31. Wu E, Robinson L, Jenny ME, Rockwood TH, Feusner J, Friedman D, Kane RL, Bhatia S: Assessment health-related quality of life of adolescent cancer patients using the Minneapolis-Manchester quality of life adolescent questionnaire. Pediatr Blood Cancer 2007, 48:678-686.

32. Hutchings HA, Upton P, Cheung WY, Maddocks A, Eiser C, Williams JG, Russell IT, Jackson S, Jenney ME: Adaptation of the Manchester-Minneapolis quality of life instrument for use in the UK population. Arch Dis Child 2007, 92:855-860.

33. Hutchings HA, Upton P, Cheung WY, Maddocks A, Eiser C, Williams JG, Russell IT, Jackson S, Jenney ME: Development of a parent version of the Manchester-Minneapolis quality of life survey for use by parents and carers of UK children: MMQL-UK (PF). Health Qual Life Outcomes 2008, 6:19.

34. Reimers TS, Mortensen EL, Nysom K, Schmiegelow K: Health-related quality of life in long-term survivor of childhood brain tumor. Pediatr Blood Cancer 2009, 53:1086-1091.

35. Einberg EL, Kadrija I, Brunt D, Nygren JN, Svedberg P: Psychometric evaluation of a Swedish version of Minneapolis-Manchester quality of life-youth form and adolescent form. Health Qual Life Outcomes 2013, $11: 79$

36. Hiraishi K: A Study on the Development of Self-Consciousness in Adolescence (I) - an examination of two dimensions of self-consciousness. Bull Fac Ed Nagoya Univ (Educational Psychology) 1990, 37:217-234 (in Japanese). 
37. Hiraishi $K$ : The structure of self-consciousness in adolescence: psychological health from a point of view of "self-establishment" and "self-diffusion". Jpn J Educ Psychol 1990, 38:320-329 (in Japanese).

38. Kline P: The Handbook of Psychological Testing. 2nd edition. London: Routledge; 1999.

39. Childhood Cancer Taskforce, Cancer Control Promotion Council, Ministry of Health, Labor and Welfare: The Proposal Document for Childhood Cancer. Tokyo: Ministry of Health, Labor and Welfare; 2011 (in Japanese).

doi:10.1186/s12955-014-0127-y

Cite this article as: Koike et al.: Development of the Japanese version of the Minneapolis-Manchester Quality of Life Survey of Health - Adolescent Form (MMQL-AF) and investigation of its reliability and validity. Health and Quality of Life Outcomes 2014 12:127.

\section{Submit your next manuscript to BioMed Central and take full advantage of:}

- Convenient online submission

- Thorough peer review

- No space constraints or color figure charges

- Immediate publication on acceptance

- Inclusion in PubMed, CAS, Scopus and Google Scholar

- Research which is freely available for redistribution 